\title{
Effects of Restorative Environments on \\ Creativity in Case of Architecture Education
}

BESTE SABIR

Istanbul Technical University

architectural education, architectural creativity, creativity, meditative spaces, neuroscience of creativity, restorative environments 
Creativity is a mental process, and cognitive psychology has focused on this subject, especially in the last century. While neuroscience concentrates on creative processes; new data emerges. When we consider architectural production as a creative process, the "free association REST thinking mode" focuses on the principle of free circulating thought, allowing relaxation and free-thinking to lead to new connections (creative moments) in the brain. The paper aims to focus on how spaces affect the creative process in case of architectural education, production, and creation. If REST mode - as relaxation, meditation, and awareness - supports the process of creation, how do restorative (calming, meditative) spaces and environments affect this process as well? With this approach, students will be questioned with quantitative methods to collect data about the effects of faculty and meditative environments on the creative process.

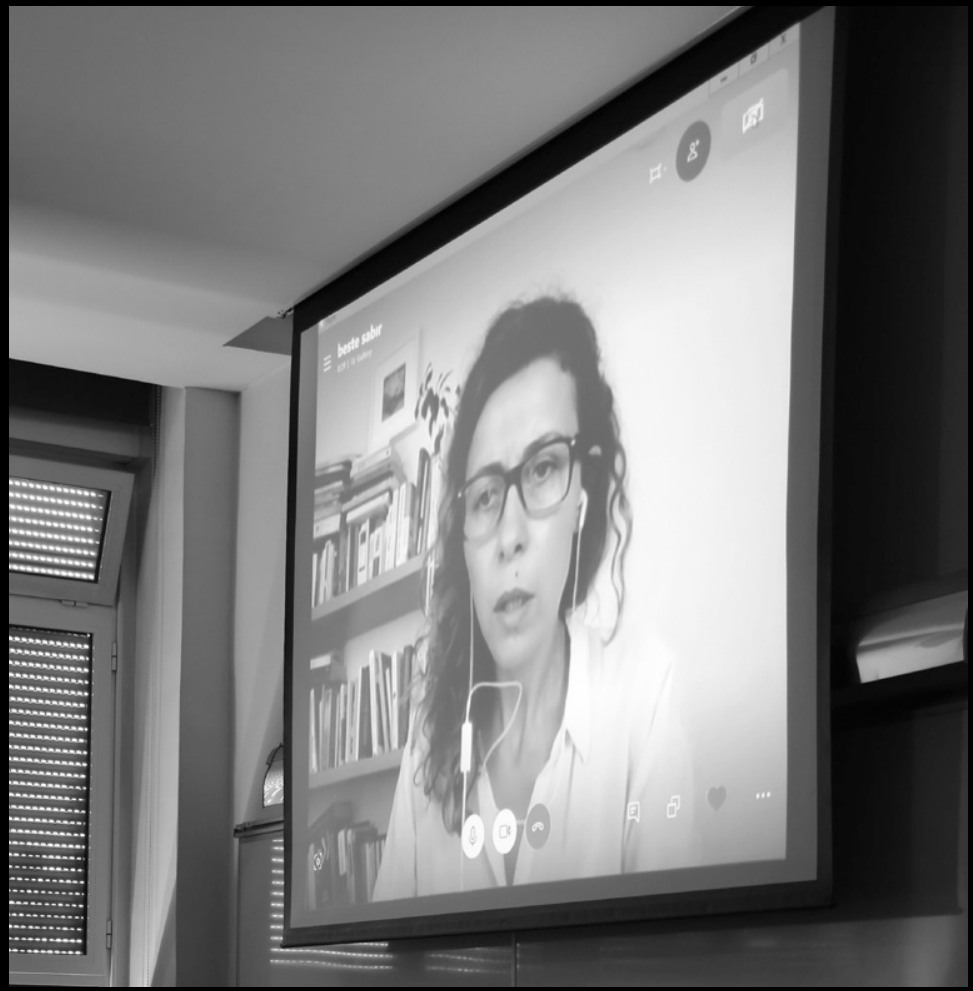




\section{INTRODUCTION}

Creativity happens when a thought comes up to surface in mind, it has a complex nature, and it does not happen in a tabula rasa condition. As Andreasen states, the interaction of human thoughts with socio-cultural situations create this phenomenon (Andreasen, 2006). Portillo defines creativity as an interconnected and multidimensional construct involving person, process, product, and place (environment/ press). The environment and a person's creativity are connected (Portillo, 1996). One of the main intentions of this paper is to address the relationship between creativity and its supportive environment in the case of architectural education, which can be defined as a design study that gets its origins from creativity. Freeman categorizes creativity with the stages such as: Preparation, Incubation, Enlightenment (A-ha moment), Affirmation/Verification (Freeman, 1971). Following that organization, we can consider that the first two stages are extraordinarily complex, intricate, and interactive processes, and in case of architectural education and its environmental necessities, designs should be answering to this complexity and interactivity as well. On the other hand, as Andreasen researches, the A-ha moment process is dependent on meditative and restorative times, and during that time the brain can be more creative and make more connections, which can become a new thought and a creative idea.

Andreasen deepens to the A-ha moment stage and even though creativity is a complex process, while the brain is in the REST (random episodic silent thought) mode, which is described as "free association", the mind is relaxed and focused onto only one subject such as breath, it creates new synapses and connections by picking up topics from the unconscious fountain of knowledge that has been collected (Andreasen, 2006). The psychologists researched with experiments such as divergent thinking questionnaires and declared that, in the REST mode, while the mind is quiet, meditative, and free-thinking, the brain generates new creative ideas.

This paper is derived from a cognitive psychology book of Andreasen that focuses on the neuroscience of creativity and the REST mode (Andreasen, 2006). With this data, the paper traces neuroscience of creativity in architectural education environments, within the relationship of REST mode, searching on the effect of meditative and restorative environments on creativity. After reaching the research about REST mode where our brain is in relaxation and meditation, our creativity is getting higher, paper askes in order to support creativity, can restorative environments play a role in creativity? 
As DeBono declares, it is important to consider creativity as the primary source in any kind of thinking and as inseparable from life itself (DeBono, 1993). Guilford describes that creativity, like many other activities, is a behavior that can be developed and learned (Guilford, 1950). This paper focuses on the neuroscience of creativity and its supportive environments that can develop this ability in architectural education.

Thus, the paper aims to ask, what is the effect of spaces to this REST mode and creative process? Can meditative and restorative environments support the REST mode and enhance creativity during architectural education? As Andreasen noted, the source of unconsciousness brings about a creation process as the result of new connections and synapsis during the free movement thought (Andreasen, 2006). Working in this sense, accepting that creativity is a complex process and that it depends on senses, observation, culture, field, stimuli, and tests, the paper aims to focus on how spaces affect the creative process in case of architectural education, production, and creation. If REST mode supports the process of creation, how do restorative (calming, meditative) spaces and environments affect this process as well?

As a case study, a questionnaire is prepared and asked to third- and fourth-year students of ITÜ Architecture Faculty, in order to get data from the results whether restorative spaces and meditative moments support their creativity in the design process and how is the creative process affected by the environment? As neuroscience declares, our environment has many effects on our behavior. Thus, to develop the creative process in architecture education, learning environments should inherit related qualities. The result of the questionnaire is expected to give feedback about architecture education environments and trying to reach the response if the meditative spaces are affecting the creative process positively.

\section{WHAT IS CREATIVITY? HISTORICAL BACKGROUND OF CREATIVITY.}

Creativity is a mental activity; as Andreasen describes, it happens when a thought comes up to surface in mind. However, this definition is missing some parts (Andreasen, 2006). Creativity does not happen away from everything in a tabula rasa environment or state of mind. The interaction of human thoughts with socio-cultural situations creates a phenomenon not only individual but also systematic scale. Tur word 'create' evolved from "creare" which means to produce, to do, and to bring into being. From the 17th century until the beginning of the 20th century, creativity was considered as equal to 
intelligence. At the beginning of the 20th century, creativity's relation to genetical factors and psychological sicknesses have been researched. Scientifically, the scientists tried to define creativity by the 20th century, with the spread of psychology as modern science. In the 1950s, scientists supported the tie between creativity and intelligence with many experiments. Moreover, by the 70s, creativity researches gained speed.

Roger MacKinnon, who researched creativity, investigated architects by using many variables, including intelligence. He was trying to reach the relation of the intelligence levels of architects with their creativity. In this research, architects have been grouped in three levels: Highly creative, creative and not creative. It can be interpreted from this phase of the research method that the researcher used interval scales for the architects to classify his variables, grouped them with his categorization on three levels. Later, he found out after the IQ tests that each architect had similar IQ scores, varying around 120. Therefore, this research declared that intelligence does not have a strong relation to creativity because the ones who are highly creative did not have any IQ score difference with the un-creative architects.

Mihaly Csikszentmihalyi stated that "creativity results from the interaction of a system composed of three elements: The first one is the knowledge field that is composing the components of culture that contain symbolic rules (Csikszentmihalyi, 1996). The second one is a field of experts who recognize and validate innovation; the third one is the individual that brings novelty into the domain. Each of the three main components affect the others and every component is necessary in creativity but not enough, in and of itself, to produce the novelty.

With Andreasen's interpretation, components of creativity are originality, utility, and the necessity of production. Therefore, generally, creativity starts with the "individual," continues by the "process," and searches the ways of conceptualizing. When it finds out the solution, the product is being formed. For cognitive neuroscience, these processes are very impressive.

Nancy Andreasen as a neuroscientist and a pioneer on cranial visualization (calculating the brain functions and qualities on living humans), developed the visualization of brain technologies. Cranial visualization is an important innovation that helps to research on human talents and brain typologies such as: How do we feel sympathy for others? How do we change the rhythm of the brain during meditation? Or, eventually, how can we benefit more from the creative talents of the brain? This visualization technique helped scientists to reach these profound questions. Andreasen researches this technique on how do acts such as feeling, thinking develop in the brain. 
For the first time in Utah Research Conference ${ }^{1}$ in 1959, researchers argued about creativity and its relationship with education, also if it can be developed. As Guilford describes, like many other activities, creativity is a behavior that can be developed and learned. Even with limited borders, creativity can be raised (Guilford, 1950).

The learning environment in which learning and creative activities take place should provide students the ground on which creativity can flourish more readily (Hasirci, 2000). There are also arguments about whether creativity is latent potentiality or an improvable characteristic (Potur, Barkul, 2006).

"We still know very little about creativity, but we know the situations that develop creative behaviors. The only thing that should be done is to bring up the potential and to be able to do that we need to release it. An individual's creativity is mostly suppressed by experiences or education; therefore, he cannot use the full potential. Nevertheless, if he is aware of himself, then he can run all the potential of his creativity" (Parnes, 1963). It can be noted that if creativity can be increased, in this process meditation and releasing creativity, restorative environments play a crucial role to enhance the mindfulness and awareness of the individual that leads to the enhancement of creativity.

\section{NATURE OF THE CREATIVE PROCESS}

Xanadu is described as some dreamy inspiration space described in a work subsequently inspiring a creative process, Samuel Taylor Coleridge's poem called Kubla Khan; or, A Vision in a Dream: A Fragment. In this poem, he metaphorically explained the creative and inspirational atmosphere as a physical space - environment. He wrote the book in the 19th century, and it is the most expressive book that has ever been written in history about creativity. The poem explained Xanadu as a space covered by the walls where the Kublai Khan builds a palace. Throughout the poem, Coleridge gives clues about creativity and expresses that it is a complex and uncertain process that is out of the control of the individual. During the '70s, creativity works have gained speed, additional to individual situations, and researchers also focused on neural conditions during the creative processes. Hence, there have been questions such as: What is the importance of individual

1 The third (1959) the University of Utah Research Conference on the Identification of Creative Scientific Talent, held at Alta, Utah, June 11-14, 1959. 
factors on creativity? How conscious is a creative process? Is it a result of a conscious try? How vital are divination and inspiration? How does creativity affect social environments? These questions have been added to the searches on conceptualizing scientific creativity researches. Therefore, researchers started to calculate the character and cognition. These studies ended up with experimental cognitive psychology.

Csikszentmihalyi in 1996 describes the creative process, not as linear but a complex structure that has been formed by overlapping of multi-layers, which has five stages. The first stage is Preparation: Becoming immersed in problematic issues that are interesting and arouse curiosity. The second one is Incubation: Ideas churn around below the threshold of consciousness. The third stage is the A-ha moment/inspiration: When the puzzle starts to fall together. The fourth stage is Evaluation: Deciding if the insight is valuable and worth pursuing. The last one is the Result: Translating the insight into its final work.

Koestler mentions Henri Poincaré's process as a mathematician while defining the creative process (Koestler, 1964). Poincare was studying Fuchsian theory for many years and got stuck in the theories. During restless nights he experienced a moment where he was not in a sleep mode; subseqjently, he articulates his theories after that release experience. Followed by these experiences Poincare defines the conditions of creativity as following: A long research process; second: attention to subconscious thoughts and senses; third: techniques and methods through which we can develop the thoughts coming from subconscious levels. Followingly, Patrick, in 1955, prepares a definition after the statements of Poincare supporting them with research and the definition of creative stages as: First: Preparation; second: Incubation; third: Inspiration/a-ha moment; last one: Verification. (Freeman, 1971: 41). Following that organization, we can consider that the first two stages are extraordinarily complex, intricate and interactive processes, and in the case of architectural education and its environmental necessities, should be answering to this complexity and interactivity as well. Figures 1 and 2 explain the related spaces to these stages.

Scientists researched what is happening during the creative process with two methods. The first one is with IQ tests and the second one is by looking into inward techniques. In this second technique, their research focuses on the mental processes during the creative act and on divergent-convergent thinking. Many psychologists use this method in creativity tests and investigate how a person responds to a specific question creatively (divergent) or how they respond with a specific response (convergent). 

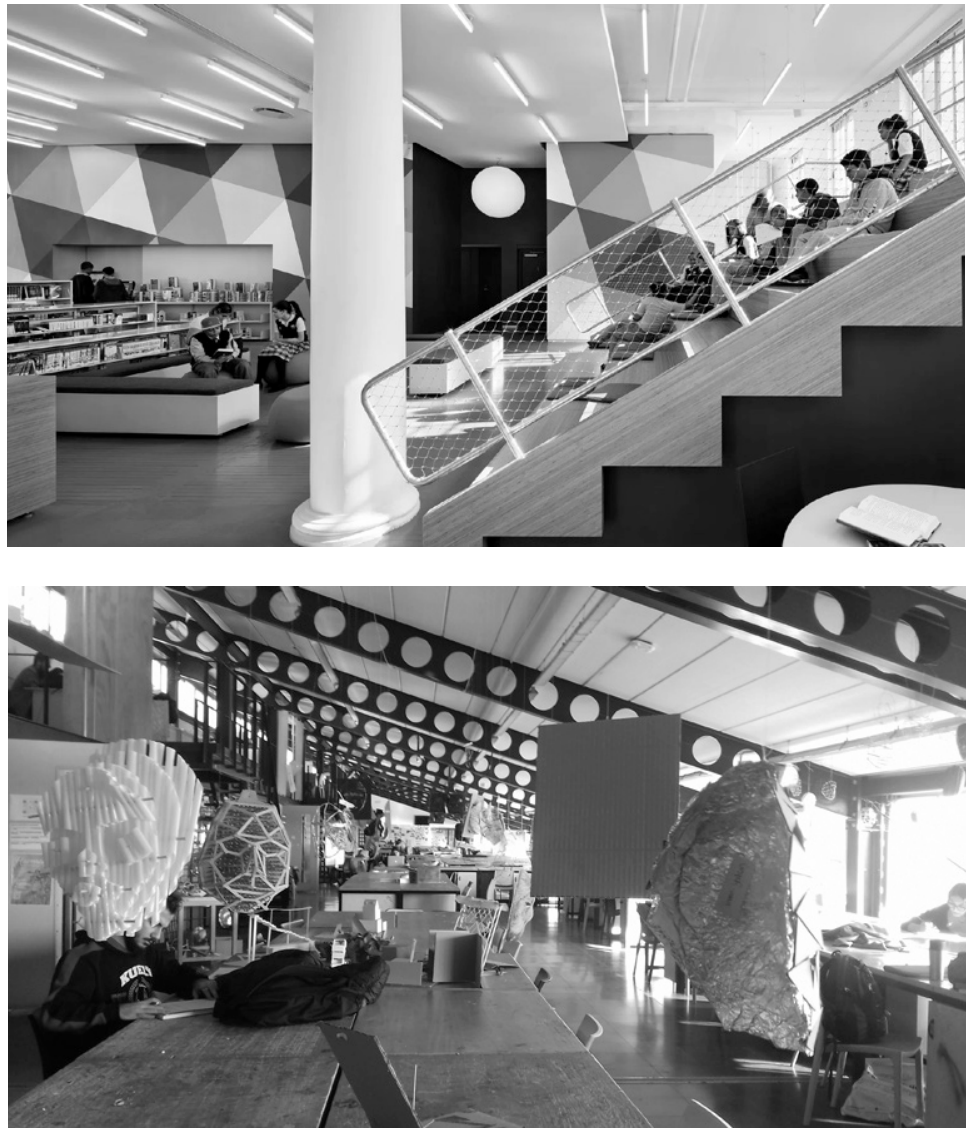

Fig. 1: Kew Gardens Hills Library, photo by Michael Moran (21.11.2019). Retrieved from https://www.curbed.com/2018/5/24/17389648/library-architecture-teens-public-space

Fig. 2: Photo from ITU, Architecture Department's design studios. Photo by the author.

Figures 1 and 2 explain the complex and interactive quality of the creative process and show the interactive environments (that support first two stages of creativity stated by Freeman); for data sharing, preparation, research, data hunting, exhibition, sharing, learning from each other which are all dynamic and intricate processes that need suitable environments such as gathering, exhibiting, sharing, experimenting.

\section{HOW DOES THE BRAIN CREATE?}

Free association is a method that has been used by many psychologists such as Freud and Wundt. It has evolved from association psychology. It is a method not focusing on events that 
are related to a chronological and conscious way but on thinking processes. Writers such as Joyce, Faulkner, Woolf used this method of "stream of consciousness" a lot. These inspiration moments can be called sleeping awake, brain-storming, or dreamy moments, where we go out of emotional inputs and we can access only by releasing, relaxing, and thinking with the flow without any conscious try, which is an essential act for creativity.

Andreasen focuses on the "stream of consciousness" technique with cranial visualization technology and searches how and where the brain creates subconscious thoughts. When it is focused on the "stream of consciousness," it can be noted that during this process the thinking process is supported by chronological memory (autobiographical memory). This memory is related to how we relate the events with our individual level and constitutes the center of self-awareness and consciousness. However, during the "stream of consciousness" the memory that is being triggered is much more interesting. Here the chronological and autobiographical side is used less. Therefore, there is a knowledge fountain or source in deep layers we cannot access by conscious acts but only with meditations, dreams, rituals, and released time-lapses, and this is an essential source for the creativity.

When Andreasen calculates the mind activities during the relaxation times, she concludes that the brain is not "empty" during these restorative times; it is very "active." This released state triggers the free-associated thought experience, and if there is no talk, there is a silent association happening. This silent free association thinking mode can be called as REST (random episodic silent thought); during this process, the brain's secondary cortices are active. This is a part of the brain; we can call it evocation cortex as well. It receives new data from frontal, parietal, and temporal lob's senses and connects these data in different and innovative new ways. Therefore, new ideas are born. The secondary cortex is one of the latest parts that has evolved in the human body. It evolves until the age of the mid twenty's, and it keeps creating new connections.

Instead, in chronology dependent memory, the brain's memory part is being activated so we remember the things we have done in daily life and we use it mostly in memorizing. These parts are not active during the REST mode. Therefore, the more the brain is free from possessions and released, the more it uses complex parts and creates new connections from the fountain of knowledge. "And that leads to a new mathematic function, a new song, a new concept design, etc. While creativity evolves, the brain makes a freer association of ideas." (Andreasen, 2006).

In REST mode -that activates the unconscious parts- many connective cortexes are related, and many synapses have been 
created where these actions transform into new ideas and new inspirations. These moments occur mostly with looking-inward moments, where the thought is not linear and sequential and where unconscious processes play a role. The association of ideas freely comes up to surface; thus, the brain starts the creative process by dissolving. It creates connections between the symbols, words, and thoughts that have not been used as connections.

\section{STAGES OF CREATIVITY}

\section{QUALITY OF SPACES}

$1^{\text {st }}$ Preparation

$2^{\text {nd }}$ Incubation

Complex, intricate, interactive

$3^{\text {rd }}$ Inspiration/A-ha moment

Restorative, meditative, releasing

$4^{\text {th }}$ Verification

Table 1. Explaining the creative stages and their differentiating qualities to define different needs. Prepared by the author, from the interpretation of Andreasen's (2006) theories and Freeman's (1971:41) creativity definition and categorization.

\section{ENVIRONMENTS THAT ENHANCE CREATIVITY}

It has been explained in the previous parts that relaxing, meditating, and releasing moments support and enhance the creativity and the REST mode. Therefore, this part asks if restorative, calming spaces can activate our creativity and REST mode? To be able to support the REST mode, what should be done in case of creative environments? Architectural education is being the focus as a creative process, and after this part, the paper focuses on how to enhance the creativity in architectural education by environmental design. How can meditative, restorative, calming spaces interact and activate our creativity during REST mode? Neuroscientists declare nowadays that we can focus on one sole object (it can be our breath or a landscape) and by free association of ideas or by meditation, we can support our brain to create, with secondary cortex, new connections from the subconscious level and tie up new ideas to each other, that come up as "inspirations".

Kaplan, Kaplan, and Ryan (1998:67) described a restorative environment as a place to rest and recuperate, and they stated that "natural settings are particularly effective for this" (pp.67). A natural landscape can produce a restorative experience and can renew a person's cognitive powers and they are described as spaces with the following characteristics: "quiet fascination; wandering in small spaces; separation from distraction; wood, stone, old; and the view from the window (Kaplan, Kaplan, \& Ryan, 1998). 


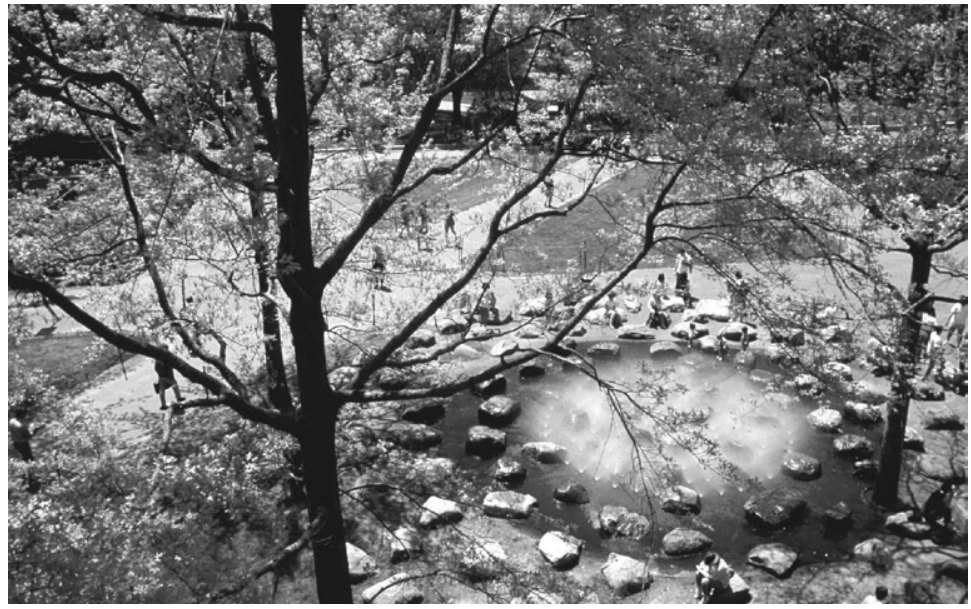

Fig. 3: Tanner Fountain at Harvard University, photo by Alan Ward. (21.11.2019). Retrieved from https://www.asla.org/awards/2008/08winners/312.html

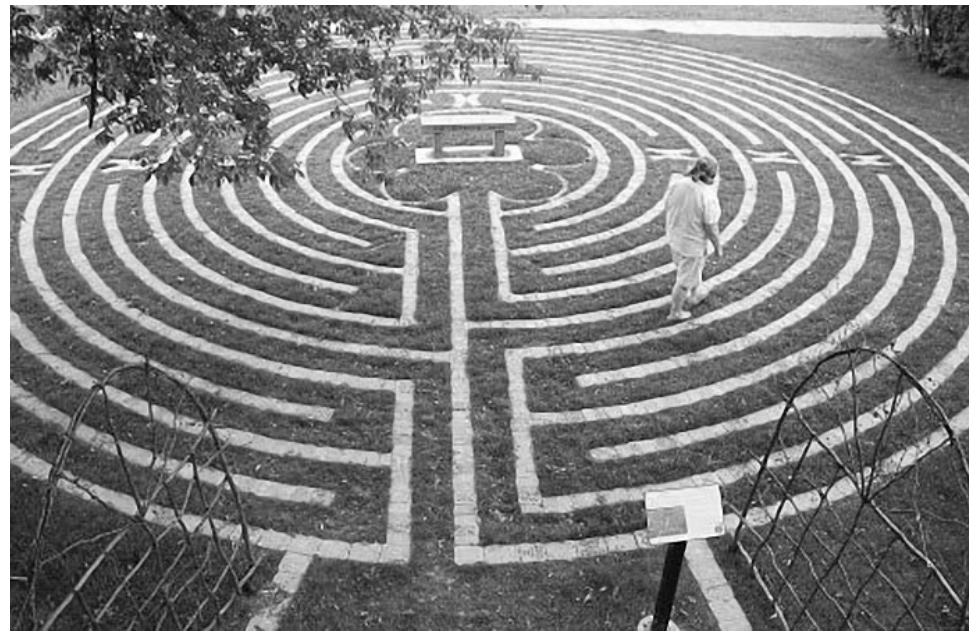

Fig. 4: Metropolitan State University, Library's Labyrinth Garden. (21.11.2019). Retrieved from http://www.bestcounselingschools.org/ best-campus-meditation-spaces/

Figures 3, 4 \& 5 refer to restorative environments that can support the REST mode by creating a meditative state and supporting the creative process in educational environments.

\section{CASE STUDY}

As a case study, twenty architecture students from third and fourth grade at the Istanbul Technical University (ITÜ) Architecture Faculty have been interviewed to form an idea if the restorative environments are supporting the creative process 


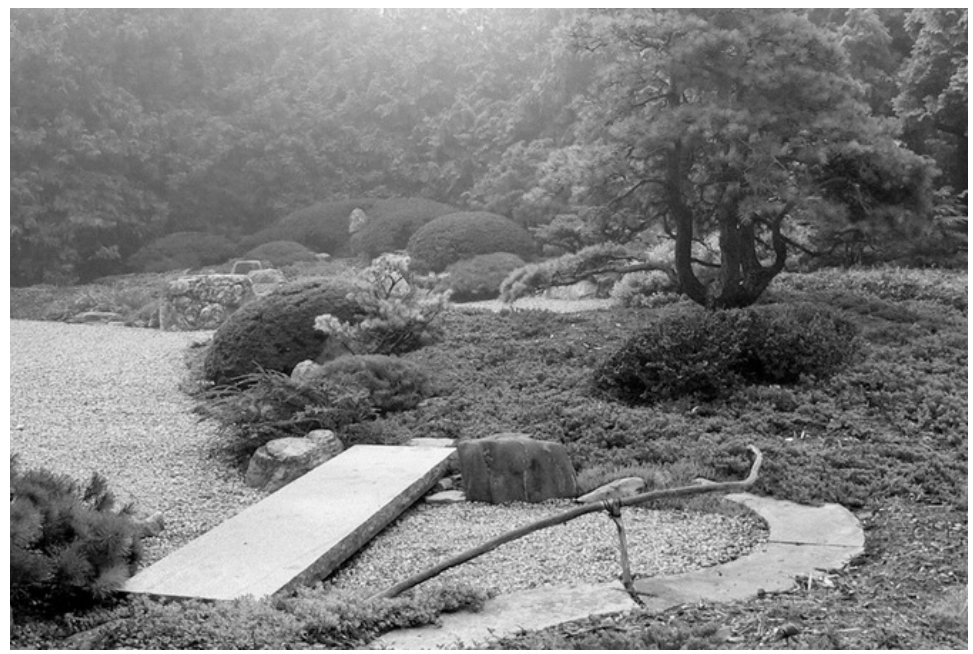

Fig. 5: Carleton College-Japanese Garden. (21.11.2019). Retrieved from http:// www.bestcounselingschools.org/best-campus-meditation-spaces/

at an inspirational level. Also, if their creative process is being affected by the environment?

As neuroscience states, our environment has many effects on our behaviors. Thus, to develop the creative process in architecture education, learning environments should inherit related qualities. During the preparation of the questionnaire, behavioral answers are expected from the students. To be able to prepare the questions, creative processes, types of each process, and categories have been researched. The questions are focusing on the students' design process, tracing their creative moments, their design project's creative moments, and the effect of the environment on this creation. The questions are constructed on the thought that the creative process needs different types of spaces because it also includes four different types of periods. While we gather the data and synthesize these data, students need very sophisticated and interactive atmospheres, but while developing and creating a new thought, what kind of spaces/environments are supporting this creative formation? There are quantitative methods to evaluate the results of this research paper. More than open-ended, mostly closed-ended questions are asked in the questionnaire.

It is kept as simple, short, understandable as possible. It has been thought that by the third year of architectural education, the creative process and understanding of this formation will be more apparent by the students.

The literature review on creativity, neuroscience of creativity helped a lot while preparing and grouping the systematic structure of the questions. In the paper, it can be expressed 
as the dependent variable; creativity, independent variables are the architectural education environment's quality.

1. If we divide the architectural design process into three parts as preparation, A-ha moment, and conclusion:

(a) Which of the following from the box below explains the necessities of the first step: preparation? (as we do the exchange of ideas, chat, communicate, research, observe, etc.)

Complexity, meeting, interaction, and playfulness have been selected by $\% 70$ of the students.

\begin{tabular}{|c|c|c|c|c|}
\hline Comfort & Meeting & $\begin{array}{l}\text { Open-wide } \\
\text { spaces }\end{array}$ & Individuality & Interaction \\
\hline Complexity & Simplicity & Calmness & Nature & Natural light \\
\hline Playfulness & Freedom & $\begin{array}{l}\text { Discipline, } \\
\text { rules }\end{array}$ & Other: & \\
\hline
\end{tabular}

(b) Which of the following from the box above explains the necessities of the second step as we call a-ha moment/inspiration? Nature, comfort, individuality, calmness, and wide spaces are being chosen by $\% 70$ of the students.

2. Until now, where/ in which kind of atmospheres did you receive creative insights, thoughts about your designs?

Quiet, comfortable, home comfort has been selected by $\% 80$ of the students. The rest of the students (\%20) have chosen the limited time, complex places.

\begin{tabular}{|l|l|l|l|}
\hline Calm quiet places & Limited places & In nature & Other \\
\hline Uncomfortable places & Crowded places & Home comfort \\
\hline
\end{tabular}

3. During your design moments when you stuck, what do you do?

I sleep, wonder, and walk, do nothing, try to relax, refresh, have been chosen by $\% 90$ of the students. Go to café, play football, socialize, get upset, try have been chosen by $\% 10$ percentage of the students. 


\begin{tabular}{|l|l|l|l|}
\hline Change the place & Go to cinema & Sleep & Read \\
\hline Try to draw & $\begin{array}{l}\text { Get upset and } \\
\text { try }\end{array}$ & $\begin{array}{l}\text { Wonder, walk and } \\
\text { do nothing }\end{array}$ & $\begin{array}{l}\text { Research exam- } \\
\text { ples }\end{array}$ \\
\hline $\begin{array}{l}\text { Refresh, shower, } \\
\text { pray, meditate, } \\
\text { try to relax }\end{array}$ & \begin{tabular}{l} 
cycle \\
\hline
\end{tabular} & $\begin{array}{l}\text { Chat, fb, go to } \\
\text { cafe }\end{array}$ & Other \\
\hline
\end{tabular}

We can notice from this question and answers that: Relaxing, refreshing, and restorative environments play a crucial role in design and creativity.

\section{CONCLUSION}

As it can be noted from the results of the questionnaire, incubation and preparation, processes are complex and need multi-actor, interactive dynamics. On the other hand, inspiration, a-ha moments depend on individuality, calmness, comfort, quietness. The creative process does not continue in a linear way; it has a complex, overlapping dynamic; that is why creative environments and, in the case of this paper, architectural education environments should be designed through this hypothesis, where the restorative environments activate our REST mode.

Design and creation have a complex system, needing(random episodic silent thought); interaction, playfulness, and restorative, meditative environments as well. Following the creativity stages and their definitions, each stage has its supportive element. During the preparation and incubation process, students should have complex, dynamic, and interactive spaces to support these stages where they learn and share. However, A-ha moment/inspiration times and the REST mode should be supported by restorative spaces and calming areas where students can activate their creative parts of the brains.

The responses of the students to the questionnaire overlap with our hypothesis.

- Incubation and preparation processes are complex and need multi-actor, interactive dynamics.

- Whereas inspiration \& a-ha moments depend on individuality, calmness, release, comfort, quietness.

- The creative process has a complex, overlapping, dynamic quality; that is why creative environments and architectural education environments might be designed through this hypothesis.

- Creativity needs both interaction, complexity, playfulness, and also restorative and meditative environments as well in order to activate the REST mode. 
Andreasen, N. (2006). The Creative Brain: The Science of Genius. Plume.

Bohm, D. (1998). On Creativity. Routledge.

Csikszentmihalyi, M. (1996). Creativity: The Psychology of Discovery and Invention. New York: Harper Collins.

Csikszentmihalyi, M. (2013). Creativity: Flow and The Psychology of Discovery and Invention. Harper Perennial.

DeBono, E. (1993). Serious Creativity: Using the Power of Lateral Thinking to Create New Ideas. London: Harper Collins Publishers.

Demirbaş, O., Demirkan, H. (2000). Privacy dimensions: a case study in the interior architecture design studio. Journal of Environmental Psychology, 20, (1), 53-64.

Freeman, J. (1971). Creativity: A Selective Review of Research, Society for Research into Higher Education Ltd, pp.41.

Guilford, J. P. (1950). Creativity. American Psychologist, 5(9), 444-454.

Hasırcı, D. (2000). The Effects of the Design and Organization of Learning Environments on Creativity: The Case of Two Sixth Grade Art-Rooms. MFA Thesis. Bilkent University.

Kaplan, R., Kaplan, S., Ryan, R (1998). With people in mind:

Design and management of everyday nature. Washington, D.C.: Island Press.

Kaufman, S., Gregoire, C. (2015). Wired to Create: Unravelling the Mysteries of the Creative Mind. TarcherPerigee.

Koestler, A. (1964). The Act of Creation. New York: Macmillan. Mallgrave, H.F. (2011). The Architect's Brain: Neuroscience, Creativity, And Architecture. Wiley-Blackwell.

Parnes, J. (1963). Education and Creativity, Teachers College Record, vol64, 1963, pp. 331-339.

Portillo, M. (1996). Uncovering implicit theories of creativity in beginning design students. Journal of Interior Design, 22(2), 16-24.

Potur, A., Barkul, A. (2006). Creative Thinking in Architectural Design Education, 1st International CIB Endorsed METU Postgraduate Conference Built Environment \& Information Technologies, Ankara.

Runco, M. (2014). Creativity: Theories and Themes: Research, Development, and Practice. Academiz Press. 


\section{APPENDIX 1:}

Questionnaire:

1. If we divide the architectural design process into three parts as preparation, A-ha moment, and conclusion:

ำ

(a) Which of the following from the box below explains the necessities of the first step: preparation? (as we do the exchange of ideas, chat, communicate, research, observe, etc.)

\begin{tabular}{|l|l|l|l|l|}
\hline Comfort & Meeting & $\begin{array}{l}\text { Open-wide } \\
\text { spaces }\end{array}$ & Individuality & Interaction \\
\hline Complexity & Simplicity & Calmness & Nature & Natural light \\
\hline Playfulness & Freedom & $\begin{array}{l}\text { Discipline, } \\
\text { rules }\end{array}$ & Other: & \\
\hline
\end{tabular}

(b) Which of the following from the box above explains the necessities of the second step as we call a-ha moment/inspiration?

2. Until now, where/ in which kind of atmospheres did you receive creative insights, thoughts about your designs?

\begin{tabular}{|l|l|l|l|}
\hline Calm quiet places & Limited places & In nature & Other \\
\hline Uncomfortable places & Crowded places & Home comfort & \\
\hline
\end{tabular}

3. During your design moments when you feel stuck, what do you do?

\begin{tabular}{|l|l|l|l|}
\hline Change the place & Go to cinema & Sleep & Read \\
\hline Try to draw & $\begin{array}{l}\text { Get upset and } \\
\text { try }\end{array}$ & $\begin{array}{l}\text { Wonder, walk and } \\
\text { do nothing }\end{array}$ & $\begin{array}{l}\text { Research exam- } \\
\text { ples }\end{array}$ \\
\hline $\begin{array}{l}\text { Refresh, shower, } \\
\text { pray, meditate, } \\
\text { try to relax }\end{array}$ & $\begin{array}{l}\text { cycle } \\
\text { Plactball, bi- }\end{array}$ & $\begin{array}{l}\text { Chat, fb, go to } \\
\text { cafe }\end{array}$ & Other \\
\hline
\end{tabular}

